REVISTA PROYECCIONES $\mathrm{N}^{\circ} 10: 141-145$

Diciembre 1985 - ISSN 0716-0917

Jornada Matemáticas, Agosto 1985.

\title{
APROXIMACIÓN LINEAL DE SEGUNDA ESPECIE
}

Ing. ERLING JOHNSON*

Dr. VICTOR VALDERRAMA*

\section{INTRODUCCION.}

Una función o aplicación Regular (finita, uniforme, continua y de rivable) será Simple cuando sus derivadas de cualquier orden, si existen, son tambiēn continuas. Las funciones secclonalmente continuas estān formadas por trozos sucesivos de aplicaciones RS y admiten una expansión en serie con respecto a una base de funciones $B_{n}(x)$. El desarrollo asi obtenido define una función analítica (que en muchos casos es RS); por eso a la expre sión primitiva (la seccionalmente continua), la llamaremos Analítica Compues ta $(\mathrm{AC})$.

\footnotetext{
* Departamento de Matemáticas y Física, Universidad de Magallanes.
} 


\section{RBSUMEN.}

La idea central que orienta nuestro trabajo es la siguiente "En su dominio de existencia las funciones AC mantienen sus RELACIONES ESENCIA LES en cualquier subintervalo finito (por pequeño que este sea)". Entende remos por RELACIONES ESENCIALES a las que permanecen invariantes del parti cular sistema de coordenadas que se emplee. Ellas expresan propiedades geométricas de la curva que representa a la función. Por ejemplo la expre sión $A^{2} \star X^{2}+B^{2} \star Y^{2}+2 \star A * B * Y+C * X+D * Y+F=0$. Podrá tener, para valo res dados de " $\mathrm{X}$ ", diversas propiedades locales tales como curvaturas, pendiente, intersección con los ejes coordenados, valores extremos etc., pero lo que será invariante de cualquier sistema usado (sea este cartesiano polar, pluckeriano u otros), es que TODOS los puntos equidistan de un punto y una recta fija. Esta propiedad de la parábola la hace inconfundible de cualquier otra curva.

Con los desarrollos en serie sucede algo análogo (los coeficientes dependerán además del sistema de coordenadas del subintervalo usado y la base elegida), pero la expansión en cualquier subintervalo mantiene las relaciones esenciales de la función tal como fueron establecidas en el intervalo primitivo. Esto último nos va a permitir reconstituir la curva a partir de cualquier trozo de ella.

En forma más concreta, hemos resuelto el siguiente problema:

"Existe una función $F(x)$ cuya expresión analítica se ignora, pero que se conocen tantos puntos de ella como se deseen en el intervalo $[b, c]$. Se determinan los valores de $F(x)$ en otro intervalo [e,h] que no incluye necesariamente $a[b, c] "$. Es más que una extrapolación porque obte nemos los VERDADEROS coeficientes de la expresión en serie en [e,h]. 


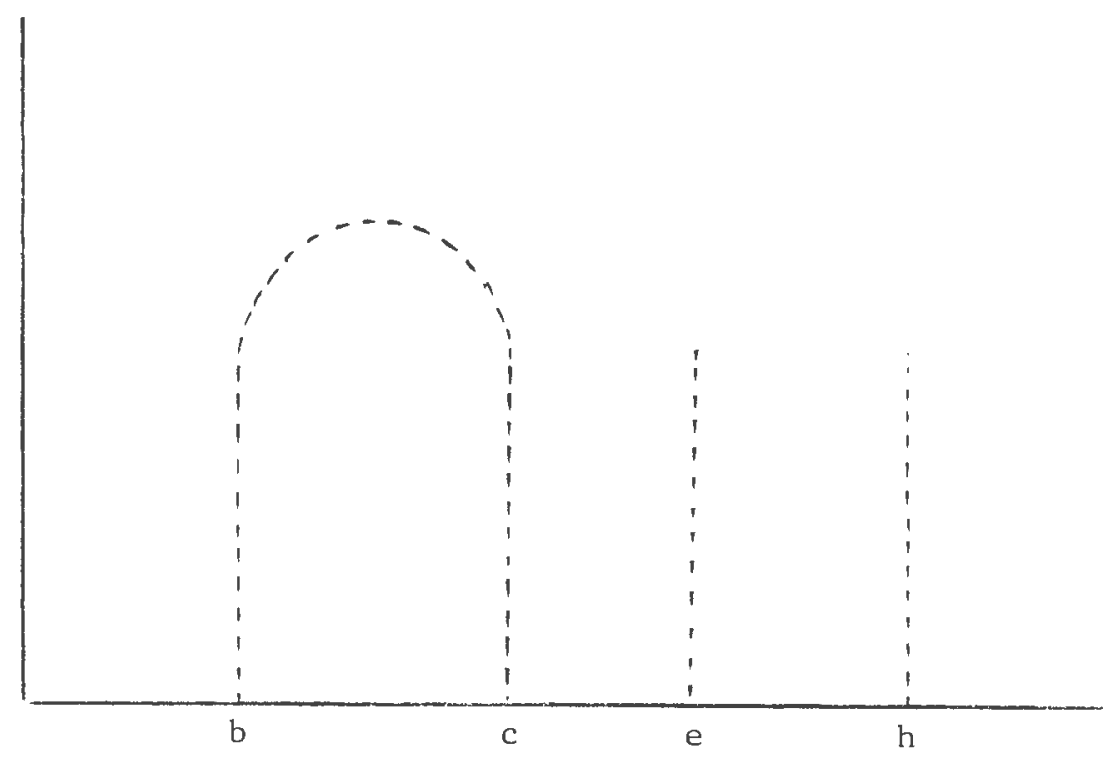

La metodología usada consistió en encontrar un operador $\{\tau\}$ que aplica $L^{2}(b, c)$ en $L^{2}(e, h)$. Como en la práctica se trabaja en el espacio

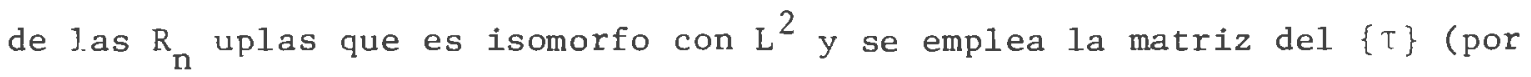
supuesto que previamente hemos definido las bases) se llega a'expresar cada coeficiente de la serie en (e,h) como una combinación lineal de los coe ficientes de la serie en $(b, c)$.

$$
I_{k}=\sum m_{k i} f_{i}
$$

Los $\mathrm{m}_{\mathrm{k}}$ son únicos y pueden ser calculados previamente ya que so 1o dependen de las bases usadas. Esta relación lineal es la que 1lamaremos "APROXIMACION LINEAL DE SEGUNDA ESPECIE". 


\section{RESULTADOS OBTENIDOS.}

- Se obtuvo una expresión para los $\mathrm{m}_{\mathrm{ki}}$ con bases de Fourier, 1imitando las funciones base a un subintervalo ( $p$ ) veces menor y coincidente en un extremo.

- Se obtuvo una matriz que permite movilizar el subintervalo en el interior del mayor, resultó ser una matriz diagonal por bloques.

- Se invirtió la matriz de Fourier con $(p)=2$ en forma explícita de una di mensión de 16 × 16 mediante el método de inversión por bloques.

- Se probó que la Aproximación Lineal es exacta para las 16 primeras funcio nes base del intervalo mayor, por ende podemos asegurar que es de la misma calidad para funciones desarrollables como una composición lineal de ellas.

- Se estudió la propagación de error que implica aplicar la transformación 1o cual demostró que la matriz es estable bajo ciertas condiciones en los errores contenidos en las constantes de entrada.

Estos resultados forman parte del informe de avance presentado en el proyecto de investigación 1984 patrocinado por la Universidad de Magalla nes, los siguientes resultados forman parte de etapas previstas en el proyec to de investigación aprobado por la Universidad de Magallanes, para 1985.

- Se obtuvo un algoritmo simple de desarrollar computacionalmente para los $m_{k i}$ con polinomios de Legendre extendidos (o sea definidos en $\{-a, a\}$ ), co mo funciones base, se probó que la matriz es triangular superior.

- Se obtuvo la matriz en forma explícita para polinomios de Legendre con una dimensión de $15 \times 15$.

- Por su estructura se obtuvo un algoritmo computacional simple para su inversión.

- Se invirtió la matriz con $(p)=2$ de $15 \times 15$.

- Se comprobó que el método opera eficientemente para funciones expresables como una composición lineal de los primeros 15 polinomios.

- Se obtuvo un algoritmo para desarrollar los $\mathrm{m}_{\mathrm{k}}$ computacionalmente cambian do de base de Fourier a Legendre y viceversa sin cambiar el intervalo. 
POR LO PRONTO PRETENDEMOS.

Estudiar las singularidades del método con funciones test en las cuales la convergencia con las bases ya vistas sea baja. 